\title{
Manual therapy versus therapeutic exercise in non-specific chronic neck pain: study protocol for a randomized controlled trial
}

\author{
Carlos Bernal-Utrera ${ }^{1 *}$ DD, Juan José González-Gerez², Manuel Saavedra-Hernandez ${ }^{3}$, \\ Miguel Ángel Lérida-Ortega ${ }^{4}$ and Cleofás Rodríguez-Blanco ${ }^{2,5}$
}

\begin{abstract}
Background: The underlying mechanisms of non-specific chronic neck pain relapses are not clear, but they could be associated with a deficit and alteration of neck muscles propioception that play a decisive role in cervical joint position, motor control of the head, and postural stability. Numerous treatments for non-specific chronic neck pain have been described in the scientific literature. However, few studies analyze its influence on postural stability, since these alterations are not fully described, and various theories emerge about the reasons that cause it. Our primary aim is to analyze the differences in postural stability, pain, cervical disability, and the relation between them produced by a treatment based on manual therapy and another based on therapeutic exercise.
\end{abstract}

Methods: The short-term and mid-term changes produced by different therapies on subjects with nonspecific chronic neck pain will be studied. The sample will be randomly divided into three groups: manual therapy, therapeutic exercise, and placebo. As dependent variables of the study, we will take (1) Overall Balance Index, measured through a dynamic stabilometric platform; (2) pain, based on the visual analog scale and the Pressure Pain Threshold; (3) cervical disability, through the neck disability index. The findings will be analyzed statistically considering a $5 \%$ significance level $(p \leq 0.05)$.

Discussion: Our study aims to provide knowledge about postural stability and its relationship with pain in subjects with non-specific chronic neck pain. Analyzing the results produced by different types of therapy will allow us to draw conclusions about the mechanisms, structural or central, that may elicit these alterations.

Trial registration: Brazilian Clinical Trials Registry, RBR-2vj7sw. Registered on 28 November 2018.

Keywords: Neck pain, Chronic pain, Exercise therapy, Musculoskeletal manipulations, Postural stability, Physical therapy specialty

\section{Background}

Non-specific neck pain is pain that does not show pathognomonic signs and symptoms [1]. When the duration of symptoms is greater than 12 weeks of evolution, it acquires the value of chronicity and is denominated nonspecific chronic neck pain (NCNP) [2]. It is a common disorder, which generates a great impact and socio-economic cost [3].

\footnotetext{
* Correspondence: cbernal495@gmail.com

${ }^{1}$ Doctoral Program in Health Sciences, University of Seville, Seville, Spain

Full list of author information is available at the end of the article
}

The underlying mechanisms of NCNP relapses are not clear, but the pain could be associated with a deficit and alteration of the proprioception of the neck muscles that play a decisive role in the cervical joint position, motor control of the head, muscles, and eyes, and postural stability (PS) [4-6].

Patients with NCNP usually have alterations in cervical proprioception and PS. They may also develop symptoms such as dizziness or vertigo [7, 8]. A recently published study shows that patients with NCNP suffer greater sensations of stunning and lack

(c) The Author(s). 2019 Open Access This article is distributed under the terms of the Creative Commons Attribution 4.0 International License (http://creativecommons.org/licenses/by/4.0/), which permits unrestricted use, distribution, and 
of proprioception than patients with benign paroxysmal vertigo [9].

Numerous studies downplay the efficacy of manual therapy and therapeutic exercise for pain reduction, cervical disability, and associated symptoms, such as dizziness [10-12]. However, there is less evidence of how these treatments, common in clinical practice, influence PS [13].

PS is highly influenced by the upper cervical spine and the suboccipital muscles, which are composed of up to 200 neuromuscular spindles per gram of muscle [14, 15]. This upper cervical segment is connected to the central nervous system (CNS), visual and vestibular apparatus, and sympathetic nervous system [16-19] in addition to cervical afferents through the cervico-ocular reflex (COR), the cervico-collic reflex (CCR), and the tonic neck reflex (TNR). The CCR activates the cervical musculature in response to stretching, maintaining good head position [20]; the COR acts through the vestibular reflex and the optokinetic reflex [21]. Finally, the TNR added to the vestibulospinal reflex achieves the maintenance of PS [22].

The alteration of this proprioceptive complex is not completely defined. Various theories have tried to explain how this system can be altered. Some studies indicate that there is a proprioceptive alteration due to sustained exposure to pain that affects PS through the CNS; these changes may be due to changes in the cortical representation and modulation of the cervical afferent contribution [23, 24]. In addition, some authors have begun to point out other psychobehavioral causes that could have a great influence on PS, such as anxiety, depression, or fear of movement $[5,6]$. We must bear in mind that these variables are present in numerous patients with NCNP [25, 26].

However, other researchers relate the loss of PS to the dysfunction of the upper cervical spine and its musculature, changes in the cervical mechanoreceptors, and the state of weakness of the musculature [27-29], but these are not necessarily associated with traumatic events, since these types of alterations have been identified among subjects with NCNP without exposure to trauma [29].

The area of dizziness of cervicogenic cause is quite unknown; there are several theories about its cause, and there is no consensus on the diagnostic criteria [30]. More research is needed about relationships between neck pain, PS, and cervicogenic dizziness.

\section{Primary objective}

The aim of our study is to compare two scientifically approved therapies for $\mathrm{NCNP}$-one treatment with a greater influence on the structural component, and the other one with a greater component on the central process-to observe differences in the PS of the subjects with NCNP.

\section{Secondary objective}

A secondary objective is to analyze the evolution of cervical pain and disability according to the treatment applied and the relationship with changes produced on PS.

\section{Hypothesis}

Experimental treatments have a greater beneficial effect on PS and pain of subjects with NCNP than sham treatment. The improvement in PS is linked to an improvement in the subject's pain.

\section{Trial design}

This study is a randomized, controlled, parallel, doubleblind, three-arm clinical trial of treatment.

\section{Methods/design}

\section{Sample selection}

Individuals with NCNP will be recruited through a text message broadcast on social networks in the city of Seville (Spain) and will be selected based on the eligibility criteria listed below. The study will take place in the facilities of the physiotherapy department of the University of Seville.

\section{Inclusion criteria}

The inclusion criteria are:

- Age 18-50 years

- Current neck pain

- Neck pain continued for at least the last 12 weeks [2].

\section{Exclusion criteria}

The exclusion criteria are:

- Irradiated neck pain

- Neck pain associated with vertigo

- Osteoporosis

- Psychological disorders

- Vertebral fractures

- Tumors

- Metabolic diseases

- Previous neck surgery

- Red flags (Night pain, severe muscle spasm, loss of involuntary weight, symptom mismatch)

- Physiotherapeutic treatment continued in the last 3 months

\section{Interventions}

The participants can only receive the assigned treatment; they cannot combine the treatment with drugs or other 
physiotherapeutic treatment. Any interference in the treatment will be grounds for exclusion.

\section{Group 1: manual therapy}

The "manual therapy" protocol will consist of three techniques based on scientific evidence for the treatment of neck pain [31-33]. These techniques represent a very close approximation to the treatment that is performed in the daily clinic, outside the research protocols.

This protocol will be applied in the three treatment sessions, one per week.

1. High thoracic manipulation on T4 [31]

2. Cervical articular mobilization $(2 \mathrm{~Hz}, 2 \mathrm{~min} \times 3$ series) [32]

3. Suboccipital muscle inhibition (3 min) [34].

\section{Group 2: therapeutic exercise}

The "therapeutic exercise" protocol will be taught to patients in the first session and should be done once a day during the 3 weeks of treatment. It will be reinforced by the physiotherapist in each of the three individual sessions.

\section{Week 1. Exercises 1 and 2:}

1. Cranio-cervical flexion (CCF) in supine position with towel in the posterior area of the neck (3 sets, 10 repetitions, $10 \mathrm{~s}$ of contraction each repetition with $10 \mathrm{~s}$ of rest)

2. CCF sitting (3 sets, 10 repetitions, $10 \mathrm{~s}$ of contraction each repetition with $10 \mathrm{~s}$ of rest)

\section{Week 2. Exercises 1, 2, 3, and 4:}

3. Co-contraction of deep and superficial neck flexors in supine decubitus (10 repetitions, $10 \mathrm{~s}$ of contraction with $10 \mathrm{~s}$ of rest)

4. Co-contraction flexors, rotators, and inclines. Patient will perfom cranial nerve flexion, while physiotherapist asks him to tilt, rotate, and look toward the same side while he opposes a resistance with his hand (10 repetitions, $10 \mathrm{~s}$ of contraction with $10 \mathrm{~s}$ of rest)

\section{Week 3. Exercises 1, 2, 3, 4, 5, and 6:}

5. Eccentric for extensors. With the patient seated, should perform cervical extension, then they must realize a CCF and then finish doing a cervical flexion (10 repetitions)

6. Eccentric for flexors. The patient will be in quadrupedal and neutral neck position. He should perform neck flexion, and then must realize a $\mathrm{CCF}$ and, maintaining that posture, extend the neck and then finally lose the CCF (10 repetitions).

\section{Group 3: sham treatment}

For the "control" protocol, the patient will be placed in the supine position, while the physiotherapist will lay his hands without therapeutic intention on the patient's neck for $3 \mathrm{~min}$, the physiotherapist will simulate the technique of suboccipital inhibition [34]. Later, with the laser pointer off, the patient will be contacted without exerting pressure for $10 \mathrm{~s}$. Patients assigned to the control group will receive treatment 1 or 2 after completing the study.

\section{Outcome measures \\ Neck Disability Index (NDI)}

The NDI is a self-assessment instrument of the specific functional status of subjects with neck pain with 10 elements, including pain, personal care, weight gain, reading, headache, concentration, work, driving, sleeping, and leisure. Each section is rated on a scale of 0 to 5, where 0 means "painless" and 5 means "the worst pain imaginable." The points obtained are added to a total score. The questionnaire is interpreted as a percentage. The disability categories for NDI are $0-8 \%$, without disability; 10-28\%, mild; 30-48\%, moderate; 50-64\%, serious; and 70-100\%, complete [35, 36].

\section{Visual analog scale (VAS) for pain}

The subjects participating in the study will indicate the intensity of their pain by means of a VAS of 100 $\mathrm{mm}$. They must signal on a horizontal line of 100 $\mathrm{mm}$ where they would place their pain, where $0 \mathrm{~mm}$ indicated "no pain" and $100 \mathrm{~mm}$ would be "the worst pain imaginable" [37].

\section{Pressure Pain Threshold (PPT)}

The PPT is recorded in newtons/square centimeter using a digital algometer (Force $\mathrm{Ten}^{\mathrm{TM}}$-Model FDX; Wagner, Greenwich, CT, USA) with a round tip surface area of $1 \mathrm{~cm}^{2}$. The measurement is taken on the spinous process of vertebra $\mathrm{C} 2$, the evaluator gradually increasing the pressure until the patient indicates through a "Yes" when the pain or discomfort appears. Three measurements are taken, obtaining an average value of these three measurements for the statistical analysis [38, 39].

\section{Overall Balance Index (OBI)}

We obtain the OBI measurement through a dynamic stabilometric platform (Balance System $^{\mathrm{Tm}}$ SD; Biodex, Shirley, NY, USA). The General Stability Test is applied at level of difficulty 4, with 1 being the most and 8 the least difficult level. The platform is free in the anterior-posterior and medial-lateral axes, and it allows one to obtain the OBI through deviations with respect to a zero point established before the test, 
with the platform stable. Two 20-s tests are performed, with $1 \mathrm{~min}$ between each test, with the score of the second test chosen for the statistical analysis. The index is calculated through the anterior-posterior and medial-lateral relationship + standard deviation $[40,41]$.

These variables will be measured in the pre-evaluation, first evaluation (week 2), second evaluation (week 4, short-term), and third evaluation (week 12, medium term). These evaluations will be carried out by an evaluator trained in these procedures, and the data will be stored in an Excel document.

\section{Participants' timeline}

A brief Standard Protocol Items: Recommendations for Interventional Trials (SPIRIT) schedule is provided in
Fig. 1, and a populated SPIRIT checklist is provided in Additional file 1.

\section{Sample size calculation}

The sample size was calculated using the Granmo calculator v.7.12. Based on the analysis of the variance of means, and estimating an alpha risk of 5\% (0.05), a beta risk of $10 \%(0.10)$, a unilateral contrast, a typical deviation of $10 \%(0.10)$, a minimum difference to detect of $9.8 \%(0.098)$ which is based as the minimum clinically important differences in OBI [42], and a rate of follow-up losses of 15\%, 10 subjects are required in each group, assuming that there are three groups. Finally, we will include 66 patients who will be divided into three groups, each group with at least 20 subjects, so as to overcome this value to assume the possible loss of follow-up.

\begin{tabular}{|c|c|c|c|c|c|c|c|c|}
\hline & \multicolumn{8}{|c|}{ STUDY PERIOD } \\
\hline & \multirow{2}{*}{$\begin{array}{c}\text { Enrolment } \\
\text { o Week }\end{array}$} & \multirow{2}{*}{$\begin{array}{c}\text { Allocation } \\
1 \text { week }\end{array}$} & \multicolumn{3}{|c|}{$\begin{array}{c}\text { Post-allocation } \\
\text { (treatment) }\end{array}$} & \multicolumn{3}{|c|}{ Follow-Up (evaluations) } \\
\hline TIMEPOINT & & & $\begin{array}{c}1 \\
W e e \\
k\end{array}$ & $\begin{array}{c}2 \\
W e e \\
k\end{array}$ & $\begin{array}{c}3 \\
\text { Wee } \\
k\end{array}$ & $\begin{array}{c}2 \\
W e e k\end{array}$ & $\begin{array}{c}4 \\
\text { Week }\end{array}$ & $\begin{array}{c}12 \\
W e e k\end{array}$ \\
\hline \multicolumn{9}{|l|}{ ENROLMENT: } \\
\hline \multirow{4}{*}{$\begin{array}{l}\text { Eligibility screen } \\
\text { Informed consent } \\
\text { Clinical Evaluation } \\
\text { and Inclusion - } \\
\text { Exclusion Criteria } \\
\text { Allocation }\end{array}$} & $\mathrm{X}$ & & & & & & & \\
\hline & $X$ & & & & & & & \\
\hline & $X$ & & & & & & & \\
\hline & & $X$ & & & & & & \\
\hline \multicolumn{9}{|l|}{ INTERVENTIONS: } \\
\hline \multirow{3}{*}{$\begin{array}{r}\text { Manual Therapy } \\
\text { Protocol } \\
\text { Therapeutic } \\
\text { Exercise Protocol } \\
\text { Sham Protocol }\end{array}$} & & & $X$ & $X$ & $X$ & & & \\
\hline & & & $X$ & $\mathrm{X}$ & $X$ & & & \\
\hline & & & $X$ & $\mathrm{X}$ & $\mathrm{X}$ & & & \\
\hline \multicolumn{9}{|l|}{ ASSESSMENTS: } \\
\hline $\begin{array}{r}\text { Demographic } \\
\text { Data }\end{array}$ & $X$ & & & & & & & \\
\hline $\begin{array}{r}\text { Neck Disability } \\
\text { Index }\end{array}$ & & & & & & $\mathrm{X}$ & $\mathrm{X}$ & $X$ \\
\hline $\begin{array}{r}\text { Visual Analog } \\
\text { Scale }\end{array}$ & & & & & & $X$ & $X$ & $\mathrm{X}$ \\
\hline $\begin{array}{r}\text { Pressure Pain } \\
\text { Threshold }\end{array}$ & & & & & & $\mathrm{X}$ & $X$ & $x$ \\
\hline $\begin{array}{r}\text { Overall Balance } \\
\text { Index }\end{array}$ & & & & & & $x$ & $X$ & $\mathrm{X}$ \\
\hline
\end{tabular}

Fig. 1 SPIRIT schedule for patient participation 


\section{Randomization}

Subjects will be divided into three groups by means of balanced randomization performed with free software (https://www.randomizer.org/). The randomization sequence will only be performed by the principal investigator and auditor.

\section{Blinding}

The evaluator and participants in the study will be blinded during the entire process.

\section{Statistical analysis}

The statistical analysis will be carried out using IBMSPSS Statistics 24 software. The normality test applied to all the variables will be the Kolmogorov-Smirnov test. For the contrast of intragroup hypotheses, Student's $t$ test for paired variables will be applied in the case of parametric distributions and Kruskal-Wallis $H$ for nonparametric distributions. For the intergroup hypothesis contrast, one-factor analysis of variance (ANOVA) will be used in the case of parametric distributions and Kruskal-Wallis $H$ for non-parametric distributions. Post hoc analysis will be obtained through Bonferroni's contrast for parametric distributions and Mann-Whitney's $U$ for non-parametric ones. Associations between pain (clinical improvement) and PS will be analyzed through Pearson's $R$ or Spearman's rho. The confidence level used will be $95 \%$ (0.05), and the power of the study will be $90 \%(0.1)$.

\section{Discussion}

This article presents a detailed description of a randomized controlled trial designed to analyze the results in terms of pain, disability, and postural stability of two types of treatments for non-specific chronic neck pain.

We intend to investigate a little-studied field such as postural stability in these subjects and to try to understand the mechanisms that may produce these alterations. We propose two types of treatments: one using manual therapy based on the structural influences of the neck, and another based on the therapeutic exercise that exerts its effect through more neurophysiological mechanisms. By observing the effects of these two therapies, we will try to analyze and gain a better understanding of the mechanisms that cause postural instability in patients with this type of pain. Our results intend to present whether the provocative mechanisms have a more structural component, or instead are caused by alterations produced at the level of the central nervous system by its sustained exposure to pain. In addition, we intend to establish relationships between clinical improvement in relation to pain with improvement in postural stability of the subjects and to analyze the differences depending on the treatment applied.
We have designed a randomized, controlled, doubleblinded clinical trial, with the aim that our study can contribute to increase scientific knowledge on this matter and initiate new lines of future research.

\section{Trial status}

This is the first and definitive protocol versión. Participants will be recruited between January and March 2019. Study completion is expected to be July 2019 .

\section{Additional file}

Additional file 1: SPIRIT Checklist. (DOC $122 \mathrm{~kb}$ )

\section{Abbreviations \\ CCF: Cranio-cervical flexion; NCNP: Non-Specific chronic neck pain; NDI: Neck Disability Index; OBI: Overall Balance Index; PPT: Pressure Pain Threshold; PS: Postural stability; VAS: Visual analog scale}

\section{Acknowledgements}

Not applicable.

\section{Authors' contributions}

CRB is the director of the project, contributed to the protocol development, provided clinical expertise, and is responsible for designing the statistical procedures. JJGG is the co-director of the project, contributed to the protocol development, and provided clinical expertise. MSH contributed to the protocol in the methodological design and provided clinical expertise. MALO contributed to the protocol in the statistical design and provided clinical expertise. CBU is the principal investigator, contributed to the concept and study design, provided clinical expertise, and developed the manuscript. All authors read and approved the final manuscript.

\section{Funding}

This trial was conducted with no external funding, and its costs have been assumed by the researchers.

\section{Availability of data and materials} Not applicable.

Ethics approval and consent to participate

This study complies with the Helsinki guidelines for human research and has been approved by the ethics committee of the University Hospital Virgen Macarena - Virgen del Rocío. The identification of each individual will remain concealed based on the ethical principles of confidentiality and privacy. All participants will receive an informed consent form with information about all treatments and the randomization process that they will approve for participation in the study. Patients assigned to the control group will receive treatment 1 or 2 after completing the study.

\section{Consent for publication}

Not applicable.

\section{Competing interests}

The authors declare that they have no competing interests.

\section{Author details}

${ }^{1}$ Doctoral Program in Health Sciences, University of Seville, Seville, Spain. ${ }^{2}$ Fisiosur I+D Research Institute, Garrucha, Almería, Spain. ${ }^{3}$ Nursing, Physiotherapy and Medicine Department, Faculty of Health Sciences, University of Almería, Almería, Spain. ${ }^{4}$ Health Sciences Department, Physiotherpy Area, Faculty of Health Sciences, University of Jaen, Jaen, Spain. ${ }^{5}$ Physiotherapy Department, Faculty of Nursing, Physiotherapy and Podiatry, University of Seville, Seville, Spain. 
Received: 12 March 2019 Accepted: 18 July 2019

\section{Published online: 09 August 2019}

\section{References}

1. Childs JD, Cleland JA, Elliott JM, Teyhen DS, Wainner RS, Whitman JM, et al. Neck pain: clinical practice guidelines linked to the International Classification of Functioning, Disability, and Health from the Orthopedic Section of the American Physical Therapy Association. J Orthop Sports Phys Ther. 2008;38(9):1e34.

2. Cohen SP. Epidemiology, diagnosis, and treatment of neck pain. Mayo Clin Proc. 2015;90(2):284-99 England.

3. Daffner SD, Hilibrand AS, Hanscom BS, Brislin BT, Vaccaro AR, Albert TJ. Impact of neck and arm pain on overall health status. Spine (Phila Pa 1976). 2003;28(17):2030-5.

4. Treleaven J. Sensorimotor disturbances in neck disorders affecting postural stability, head and eye movement control. Man Ther. 2008;13(1):2-11.

5. Grande-Alonso M, Moral Saiz B, Mínguez Zuazo A, Lerma Lara S, La Touche R. Biobehavioural analysis of the vestibular system and posture control in patients with cervicogenic dizziness. A cross-sectional study. Neurol (English Ed). 2018;33(2):98-106.

6. Cuenca-Martínez F, Bartrina-Rodríguez I, Suso-Martí L, La R, Ferrer-Peña R, Bartrina-Rodríguez I, et al. Association between somatosensory, motor and psychological variables by levels of disability in patients with cervicogenic dizziness. Somatosens Mot Res. 2018;0(0):1-6.

7. Treleaven J, Jull G, Sterling M. Dizziness and unsteadiness following whiplash injury: characteristic features and relationship with cervical joint position error. J Rehabil Med. 2003;35(1):36-43.

8. Kristjansson E, Treleaven J. Sensorimotor function and dizziness in neck pain: implications for assessment and management. J Orthop Sport Phys Ther. 2009;39(5):364-77.

9. L'Heureux-Lebeau B, Godbout A, Berbiche D, Saliba I. Evaluation of paraclinical tests in the diagnosis of cervicogenic dizziness. Otol Neurotol. 2014;35(10):1858-65.

10. Vincent K, Maigne J, Fischhoff C, Lanlo O, Dagenais S. Systematic review of manual therapies for nonspecific neck pain. Joint Bone Spine. 2013;80(5): 508-15.

11. Gross AR, Paquin JP, Dupont G, Blanchette $S$, Lalonde P, Cristie T, et al Exercises for mechanical neck disorders: a Cochrane review update. Man Ther. 2016;24:25-45.

12. Miller J, Gross A, D'Sylva J, Burnie SJ, Goldsmith CH, Graham N, et al. Manual therapy and exercise for neck pain: a systematic review. Man Ther. 2010; 15(4):334-54.

13. Holt KR, Haavik H, Elley CR. The effects of manual therapy on balance and falls: a systematic review. J Manip Physiol Ther. 2012;35(3):227-34.

14. Boyd L, Briggs $C$, et al. Muscle spindle distribution, morphology, and density in the longis colli and multifidus muscles of the cervical spine. Spine. 2002; 27(7):694-701.

15. Bolton PS, Kerman IA, et al. Influences of neck afferents on sympathetic and respiratory nerve activity. Brain Res Bull. 1998;47(5):413-9.

16. Selbie WS, Thomson DB, et al. Suboccipital muscles in the cat neck: morphometry and histochemistry of the rectus capitis muscle complex. J Morphol. 1993;216(1):47-63.

17. Hellstrom F, Roatta $S$, et al. Responses of muscle spindles in feline dorsal neck muscles to electrical stimulation of the cervical sympathetic nerve. Exp Brain Res. 2005;165(3):328-42.

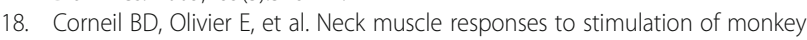
superior colliculus. I. Topography and manipulation of stimulation parameters. J Neurophysiol. 2002;88(4):1980-99.

19. Peterson BW. Current approaches and future directions to understanding control of head movement. Prog Brain Res. 2004;143:369-81.

20. Mergner T, Schweigart G, et al. Eye movements evoked by proprioceptive stimulation along the body axis in humans. Exp Brain Res. 1998;120(4):450-60.

21. Yamagata $Y$, Yates BJ, et al. Participation of la reciprocal inhibitory neurons in the spinal circuitry of the tonic neck reflex. Exp Brain Res. 1991;84(2):461-4.

22. Pera D, Graven-Nielsen T, et al. Inhibition of motor system excitability at cortical and spinal level by tonic muscle pain. Clin Neurophysiol. 2001; 112(9):1633-41.

23. Flor $\mathrm{H}$. Cortical reorganisation and chronic pain: implications for rehabilitation. J Rehab Med. 2003;35:66-72.
24. Falla D. Unravelling the complexity of muscle impairment in chronic neck pain. Man Ther. 2004;9(3):125-33.

25. Pool JJM, Ostelo RWJG, Knol D, Bouter LM, de Vet HCW. Are psychological factors prognostic indicators of outcome in patients with sub-acute neck pain? Man Ther. 2010;15(1):111-6.

26. Sarig Bahat H, Weiss PL, Sprecher E, Krasovsky A, Laufer Y. Do neck kinematics correlate with pain intensity, neck disability or with fear of motion? Man Ther. 2014;19(3):252-8.

27. Uhlig $Y$, Weber BR, et al. Fiber composition and fiber transformation in neck muscles of patients with dysfunction of the cervical spine. J Orthop Res. 1995;13:240-9.

28. MCPartland JM, Brodeur RR, et al. Chronic neck pain, standing balance, and suboccipital muscle atrophy-a pilot study. J Manip Physiol Ther. 1997;20(1):24-9.

29. Field S, Treleaven J, et al. Standing balance: a comparison between idiopathic and whiplash induced neck pain. Man Ther. 2007;13(3):183-91.

30. Reneker JC, Moughiman MC, Cook CE. The diagnostic utility of clinical tests for differentiating between cervicogenic and other causes of dizziness after a sports-related concussion: an international Delphi study. J Sci Med Sport. 2015;18(4):366-72

31. Saavedra-Hernandez M, Arroyo-Morales M, Cantarero-Villanueva I, Fernandez-Lao C, Castro-Sanchez AM, Puentedura EJ, et al. Short-term effects of spinal thrust joint manipulation in patients with chronic neck pain: a randomized clinical trial. Clin Rehabil. 2013;27(6):504-12 England.

32. Lopez-Lopez A, Alonso Perez JL, Gonzalez Gutierez JL, La Touche R, Lerma Lara $\mathrm{S}$, Izquierdo $\mathrm{H}$, et al. Mobilization versus manipulations versus sustain apophyseal natural glide techniques and interaction with psychological factors for patients with chronic neck pain: randomized controlled trial. Eur J Phys Rehabil Med. 2015;51(2):121-32.

33. Jeong E-D, Kim C-Y, Kim S-M, Lee S-J, Kim H-D. Short-term effects of the suboccipital muscle inhibition technique and cranio-cervical flexion exercise on hamstring flexibility, cranio-vertebral angle, and range of motion of the cervical spine in subjects with neck pain: a randomized controlled trial. J Back Musculoskelet Rehabil. 2018;31(6):1025-34.

34. Heredia-Rizo AM, Pascual-Vaca AO, Cabello MA, et al. Immediate effects of the suboccipital muscle inhibition technique in craniocervical posture and greater occipital nerve mechanosensitivity in subjects with a history of orthodontia use: a randomized trial. J Manip Physiol Ther. 2012;35:446-53.

35. Cleland JA, Fritz JM, Whitman JM, Palmer JA. The reliability and construct validity of the Neck Disability Index and patient specific functional scale in patients with cervical radiculopathy. Spine. 2006;31:598-602.

36. Kovacs FM, Bagó J, Royuela A, Seco J, Giménez S, Muriel A, et al. Psychometric characteristics of the Spanish version of instruments to measure neck pain disability. BMC Musculoskelet Disord. 2008;9:42.

37. Price DD, McGrath PA, Rafii A, Buckingham B. The validation of visual analogue scales as ratio scale measures for chronic and experimental pain. Pain. 1983;17:45-56.

38. Fischer AA. Algometry in diagnosis of musculoskeletal pain and evaluation of treatment outcome: an update. J Muscoskel Pain. 1998;6:5-32.

39. Chesterton LS, Sim J, Wright CC, Foster NE. Interrater reliability of algometry in measuring pressure pain thresholds in healthy humans, using multiple raters. Clin J Pain. 2007:23(9):760e6.

40. Parraca JA, Olivares PR, Carbonell-Baeza A, Aparicio VA, Adsuar JC, Gusi N. Test-retest reliability of biodex balance SD on physically active old people. $J$ Hum Sport Exerc. 2011;6(2):444-51.

41. Arnold BL, Schmitz RJ. Examination of balance measures produced by the biodex stability system. J Athl Train. 1998;33(4):323-7.

42. Salavati M, Akhbari B, Takamjani IE, Bagheri H, Ezzati K, Kahlaee AH. Effect of spinal stabilization exercise on dynamic postural control and visual dependency in subjects with chronic non-specific low back pain. J Bodyw Mov Ther. 2016;20(2):441-8.

\section{Publisher's Note}

Springer Nature remains neutral with regard to jurisdictional claims in published maps and institutional affiliations. 